\title{
Solving the problem of personnel shortage through the optimization of psychiatric education
}

\author{
Tatiana Galako, Kyrgyz State Medical Academy, Bishkek, Kyrgyz Republic
}

\section{Problem statement}

Despite the active process of the psychiatric service reform in the Kyrgyz Republic, a significant shortage of personnel still remains. The psychiatrist density is 0.3 practitioners per 10000 of the population that indicates a great need for more specialists. "Government Mental Health Care Program of Kyrgyz Republic for 2018-2030" has developed a policy of measures aimed at optimization of mental health care staffing at all levels of public health service.

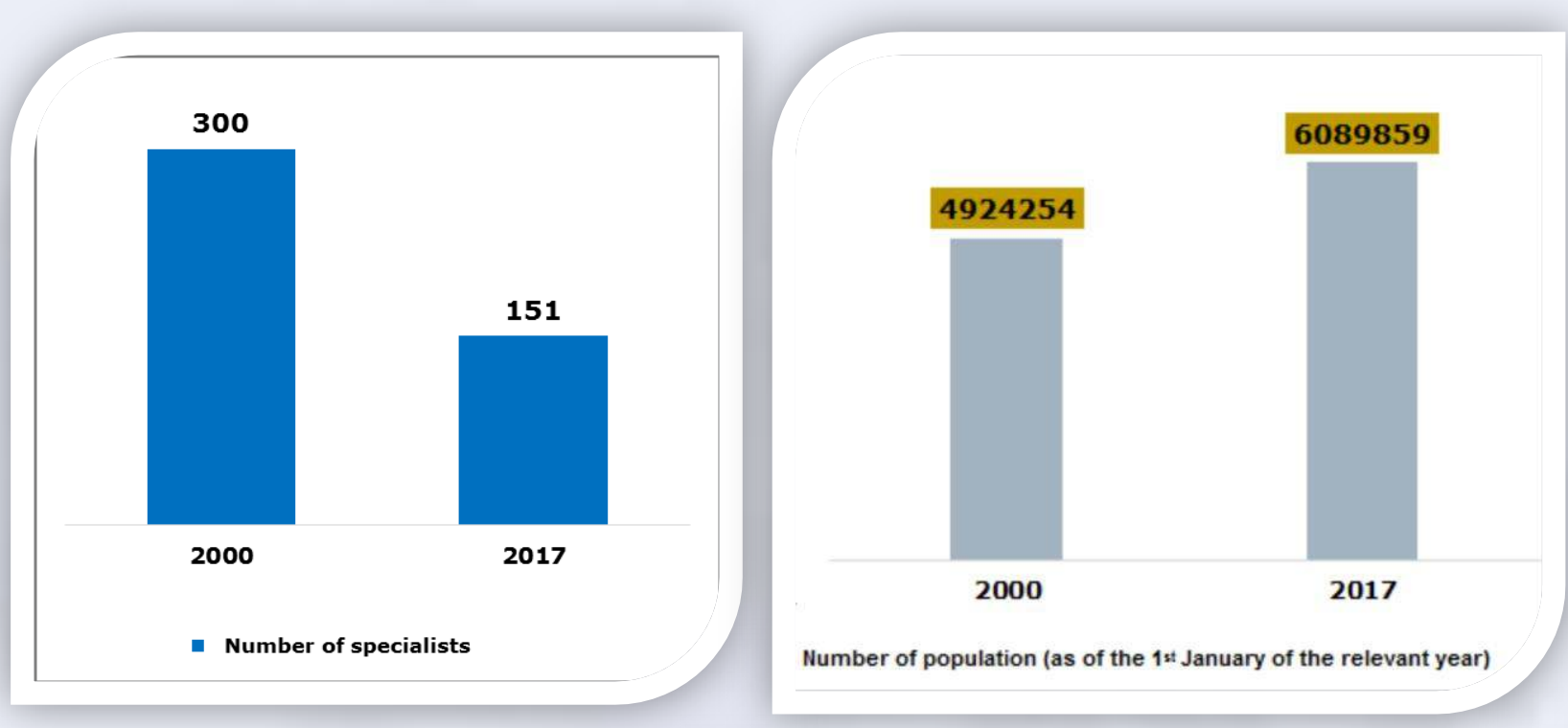

The medical institutions graduates are not motivated to get specialties of psychiatric field. Thus, among 1342 graduates of internship and clinical residency of 2016, only 5 people graduated with the major - "psychiatrist"

- While need of 3300 family doctors, only1706 are working , and more than $60 \%$ of them are pensioners. Beside the specialists of psychological and psychiatric field, family doctors are supposed to render a psychiatric aid at the first level of healthcare.

-Imperfection of educational programs on mental health problems at the undergraduate, postgraduate and continuing levels of medical education.

- The results of the research, held with the support of European regional bureau of $\mathrm{WHO}$, showed, that only $28 \%$ of family doctors have studied short term courses on mental health problems, what explains the low percent of mental disorders detection at the first level of healthcare

- Insufficient level of preparation of mental health service and initial medical branch on mental health issues

State Program on mental health care in the Kyrgyz Republic for 2017 - 2030

The problem of personnel shortage in the mental health service has already been considered at the state level. In particular, the following is envisaged: "Improvement of education in the field of mental health at the undergraduate, postgraduate and continuing levels of medical education".

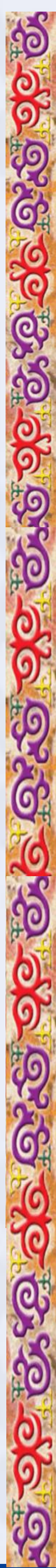

\section{Situational analysis and its results}

Situational analysis of the resources of psychiatric care at the primary health care level was carried out to develop an action plan in this direction which showed positive trends in this direction:

1) Significant changes in the system of providing psychiatric care aimed at improving its quality, approaching the patient's place of residence and the prevalence of psychosocial services

2) More than half of family doctors were trained on mental health problems, after which the indicator of detectable mental disorders at the primary level of health increased

3) Psychiatric rooms from the Mental Health Centers were transferred to the Family Medicine Centers

4) Development of new and revision of obsolete standards and clinical guidelines for mental health care at the level of primary health care.

\section{Solutions}

In order to integrate psychiatry into the primary health care network, some of the psychiatrist's functions are transferred to family doctors. More than 700 family doctors have been trained in the basics of providing psychiatric care, that has led to a significant increase in the detection of mental disorders.
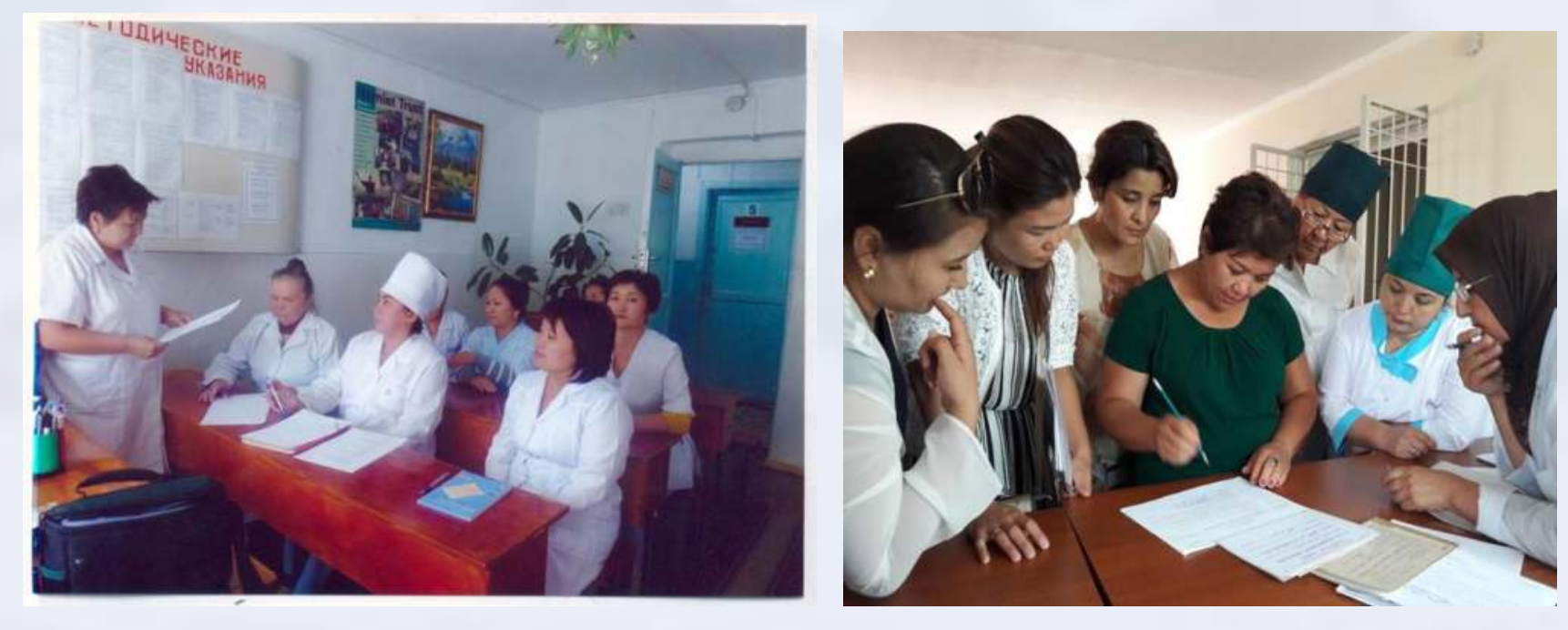

- Sustained efforts are being made to train nurses in working with patients suffering from mental disorders.

- Reforms in education are being implemented at the pre- and postgraduate levels.

- New standards and clinical guidelines are being actively developed (in the last 2 years more than 20 clinical guidelines for various levels of healthcare).

- Since 2016, a new model for the provision of comprehensive medical services has been integrated at the primary level of health care in the form of multidisciplinary teams (MDT), which provide a wide range of services (medical, psychological, social). The teams include various specialists: psychiatrists, psychologists, nurses, social workers, who have undergone special training. 